\title{
Frustrated double ionization in two-electron triatomic molecules
}

\author{
A. Chen, ${ }^{1}$ H. Price, ${ }^{1}$ A. Staudte, ${ }^{2}$ and A. Emmanouilidou ${ }^{1}$ \\ ${ }^{1}$ Department of Physics and Astronomy, University College London, Gower Street, London WC1E 6BT, United Kingdom \\ ${ }^{2}$ Joint Laboratory for Attosecond Science, University of Ottawa and National Research Council, \\ 100 Sussex Drive, Ottawa, Ontario, Canada K1A 0R6
}

(Received 22 April 2016; published 7 October 2016)

\begin{abstract}
Using a semiclassical model, we investigate frustrated double ionization (FDI) in $\mathrm{D}_{3}{ }^{+}$, a two-electron triatomic molecule, when driven by an intense, linearly polarized, near-infrared $(800 \mathrm{~nm})$ laser field. We compute the kinetic energy release of the nuclei and find a good agreement between experiment and our model. We explore the two pathways of FDI and show that, with increasing field strength, over-the-barrier ionization overtakes tunnel ionization as the underlying mechanism of FDI. Moreover, we compute the angular distribution of the ion fragments for FDI and identify a feature that can potentially be observed experimentally and is a signature of only one of the two pathways of FDI.
\end{abstract}

DOI: 10.1103/PhysRevA.94.043408

\section{INTRODUCTION}

The nonlinear response of multicenter molecules to intense laser fields is a fundamental problem, posing challenges to theory and experiment alike. The starting point of these interactions is often tunnel ionization in the laser field. Due to the oscillating electric field the ionized electron does not always escape and can be recaptured by the parent ion into a Rydberg state at the end of the laser pulse. This mechanism is called frustrated ionization [1-7]. Frustrated ionization was initially studied in $\mathrm{He}$ [2] and $\mathrm{H}_{2}$ [1], and is also a candidate for the inversion of neutral $\mathrm{N}_{2}$ in the context of free-space air lasing [8]. So far, $\mathrm{D}_{3}{ }^{+}$and $\mathrm{H}_{3}{ }^{+}$are the only multicenter molecules where frustrated ionization has been studied in a benchmark experiment $[7,9,10]$ and discussed using classical models [11,12].

Here, we explore double ionization (DI) and frustrated double ionization (FDI) of strongly driven $\mathrm{D}_{3}{ }^{+}$. In FDI both electrons ionize but one is recaptured. We model these mechanisms using a three-dimensional (3D) semiclassical trajectory simulation. Our model accounts for tunnel ionization during propagation, thus providing an improved description of FDI-a significant process that accounts for roughly $10 \%$ of all events in triatomic molecules [11,12].

Classical and semiclassical models are essential in understanding the fragmentation dynamics in triatomic molecules driven by intense infrared laser pulses. One reason is that the strongly driven dynamics of two electrons and three nuclei poses an immense challenge for fully $a b$ initio quantum mechanical calculations. Currently, quantum mechanical techniques can only address one electron in triatomic molecules in two dimensions [13]. Our work employs a 3D semiclassical model, which has provided significant insights into the FDI process for strongly driven $\mathrm{H}_{2}[5,6]$. Here, we generalize our model to triatomic molecules. We show that our result for the distribution of the kinetic energy release (KER) for FDI is in good agreement with the experimental result in Ref. [9]. Moreover, even though FDI is generally associated with tunnel ionization, we show that for increasing field strengths the mechanism underlying FDI is over-the-barrier ionization instead. We have previously shown that two pathways contribute to FDI [5]. We show that for the strongly driven triatomic $\mathrm{D}_{3}{ }^{+}$, one of the two pathways of FDI has a signature in the angular distribution of the ion fragments with respect to the direction of the laser field. Very importantly, this trace can potentially be observed experimentally.

\section{MODEL}

In our model we employ a laser field of the form

$$
\begin{aligned}
\mathbf{E}(t) & =E_{0}(t) \cos (\omega t) \hat{z} \\
E_{0}(t) & =\left\{\begin{array}{lc}
E_{0} & 0 \leqslant t<10 T \\
E_{0} \cos ^{2} \frac{\omega(t-10 T)}{8} & 10 T \leqslant t \leqslant 12 T,
\end{array}\right.
\end{aligned}
$$

with $E_{0}(t), T$, and $\omega$ the envelope, the period, and the frequency, respectively, of the laser field. We take $\omega$ equal to 0.057 atomic units (a.u.) $(800 \mathrm{~nm})$. In the following, we consider only two cases of planar alignment, i.e., one side of the equilateral, molecular triangle is either parallel or perpendicular to the $\hat{z}$ axis. Atomic units are used throughout this work unless otherwise indicated.

To compare with the experimental results [7,9] we take the initial state of the $\mathrm{D}_{3}{ }^{+}$molecule to be the one created via the reaction $\mathrm{D}_{2}+\mathrm{D}_{2}{ }^{+} \rightarrow \mathrm{D}_{3}{ }^{+}+\mathrm{D}$ [7,9]. This initial state consists of a superposition of vibrational states $v=1-12$ $[9,14]$, each with a triangular configuration. Tunnel ionization is very sensitive to variations of the ionization potential and is known to preferentially ionize larger internuclear separations $[15,16]$. Thus, we assume that most of the $\mathrm{D}_{3}{ }^{+}$ ionization occurs at the outer classical turning point of the vibrational levels. The turning point varies from 2.04 a.u. $(v=1)$ to 2.92 a.u. $(v=12)[14,17]$. We find the first and second ionization potentials of the relevant 12 vibrational states using the quantum chemistry software MOLPRO [18]. For the initial state of $\mathrm{D}_{3}{ }^{+}$in the laser field, we assume that one electron (electron 1) escapes either by tunneling or over-thebarrier ionization in the field-lowered Coulomb potential [6], depending on the field strength and the vibrational state. We use a tunneling rate given by the semiclassical formula in Ref. [19]. If electron 1 escapes by tunneling, then its transverse to the laser field velocity distribution is a Gaussian [20], while its velocity parallel to the laser field is assumed to be zero. This assumption has been recently verified experimentally for 
strongly driven $\operatorname{Ar}$ [21]. We assume that the other electron is initially bound (electron 2). Its initial state is described by a microcanonical distribution which we formulated very recently [22] and is applicable to any one-electron triatomic molecule. Since an initial predissociation does not significantly modify the ionization dynamics [6], we simplify our model by initializing the nuclei at rest for all vibrational levels.

For the propagation of our model system we generalized the technique we developed in the context of FDI of $\mathrm{H}_{2}$. Details can be found in Ref. [6]. It is worth emphasizing a feature of our model: we use the Wentzel-Kramers-Brillouin approximation to allow for tunneling of each electron during the propagation [5,6]. This is essential in order for our model to accurately describe the enhanced ionization process (EI) [23,24]. In EI, at a critical distance of the nuclei, a double potential well is formed such that it is easier for an electron bound to the higher potential well to tunnel to the lower potential well and subsequently ionize. We also note that our model fully accounts for the Coulomb singularity [6].

\section{RESULTS AND DISCUSSION}

We now consider DI and FDI of $\mathrm{D}_{3}{ }^{+}$. DI refers to the formation of three $\mathrm{D}^{+}$ions and two escaping electrons. FDI refers to the formation of a neutral excited fragment $\mathrm{D}^{*}$, two $\mathrm{D}^{+}$ions, and one escaping electron. Previous experiments on strong-field ionization of $\mathrm{D}_{3}{ }^{+}$studied, among other observables, the kinetic energy release (KER), i.e., the sum of the kinetic energies of the ion fragments [9]. To be able to compare the experimental KER with the KER from our simulation, we need to account for the intensity averaging in the focal volume.

We first compute the KER distribution for a process $\alpha=$ FDI,DI as a function of the laser field intensity as follows:

$$
P^{\alpha}(I, K E R)=\frac{\sum_{v, \phi_{0}} P_{v} P^{\alpha}\left(\phi_{0}, v, I, K E R\right) \Gamma\left(\phi_{0}, v, I\right)}{\sum_{v, \phi_{0}} P_{v} \Gamma\left(\phi_{0}, v, I\right)},
$$

where $P^{\alpha}\left(\phi_{0}, v, I, K E R\right)$ is the probability to obtain a KER from a vibrational state $v$, for an initial phase of the laser field $\phi_{0}=\omega t_{0}$ and for a laser field intensity $I . I=1 / 2 c \epsilon_{0} E_{0}^{2}$, where $c$ is the speed of light and $\epsilon_{0}$ is the vacuum permittivity. The initial phase $\phi_{0}$ corresponds to the starting point of the propagation. $\Gamma\left(\phi_{0}, v, I\right)$ is the tunnel-ionization rate computed using the semiclassical formula in Ref. [19], and $P_{v}$ is the percentage of the vibrational state $v$ in the initial state produced following the reaction generating $\mathrm{D}_{3}{ }^{+}$[14] .

Following the formulation in [25], we compute the KER distribution for a laser peak intensity $I_{\max }$ as follows:

$$
P^{\alpha}\left(I_{\max }, K E R\right)=\int_{0}^{I_{\max }} \frac{P^{\alpha}(I, K E R)}{I} d I .
$$

In practice, in Eq. (3) we integrate only over the intensities which significantly contribute to the process $\alpha$. To find the lower limit of these intensities we compute the ionization probability for an intensity $I$ and a vibrational state $v$, which for small values of the ionization probability is given by

$$
\Gamma(v, I) \approx \int_{t_{i}}^{t_{f}} \Gamma(\omega t, v, I) d t,
$$

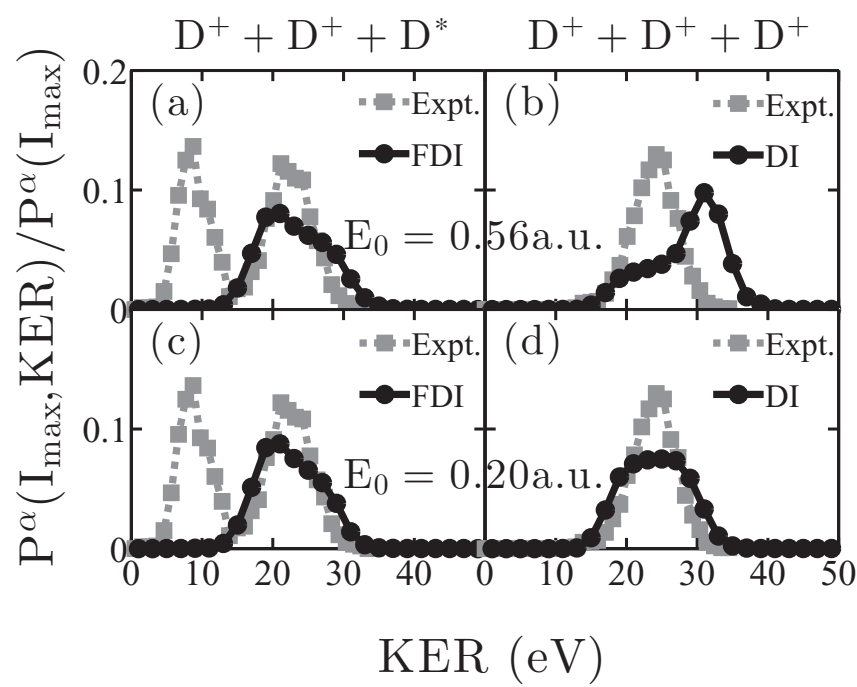

FIG. 1. Intensity-averaged KER distributions for FDI and DI for $I_{\max }$ corresponding to a field strength of $E_{0}=0.56$ a.u., (a) and (b), and of $E_{0}=0.2$ a.u., (c) and (d). The gray dashed lines show the relevant experimental results from [9]. Our results and the experimental ones for DI and FDI have been normalized to 1. Note that the experimental results in (a) and (c) have two peaks; it is the area under the higher-energy peak that has been normalized to 1 .

with the integration over the duration of the laser pulse. For the laser pulse and all the vibrational states of the triatomic we currently consider, we find that the ionization probability of $\mathrm{D}_{3}{ }^{+}$is very small for field strengths less than 0.06 a.u. Therefore, only field strengths above 0.06 a.u. contribute to the observed KER distributions.

We now compare the intensity-averaged KER distributions with the measured ones [9] for a peak field strength of 0.56 a.u., which corresponds to the experiment's intensity of $1.1 \times 10^{16}$ $\mathrm{W} / \mathrm{cm}^{2}$ [9]. We find that the KER distributions for FDI and DI for both considered alignments of the molecule relative to the laser field polarization are very similar. We therefore expect that any other planar alignment of molecule and laser field polarization will not significantly change the KER distributions. We plot the KER in Fig. 1 only for the parallel alignment. Since ionization processes can be influenced by the shape of the laser field [26,27], we have computed the KER for parallel alignment also for a Gaussian envelope laser field. We find that the shape of the KER plotted in Fig. 1 using the laser field in Eq. (1) is in complete agreement with the shape of the KER using a Gaussian envelope laser field. We find that for FDI the computed KER distribution is in good agreement with the experimental one [see Fig. 1(a)]. Both distributions peak at $21 \mathrm{eV}$, while the computed KER distribution has a wider tail towards higher field strengths. In the experimental data of the single ionization channel [Figs. 1(a) and 1(c)] an additional peak at $\sim 8 \mathrm{eV}$ is present. This peak is likely due to the bond softening [28] of an intermediate $\mathrm{D}_{2}{ }^{+}$in the experiment. Our model does not include this mechanism and hence does not show this peak.

The agreement is not as good for the KER distribution for DI shown in Fig. 1(b): the computed distribution peaks at $31 \mathrm{eV}$ while the experimental one peaks at $24 \mathrm{eV}$. It is 

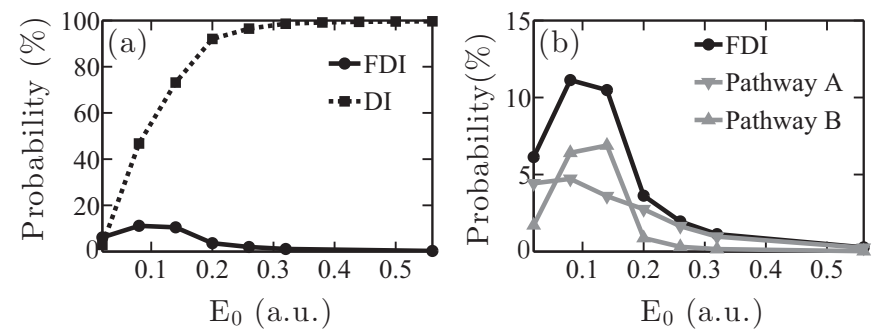

FIG. 2. The probability (a) for FDI and DI and (b) for pathways $\mathrm{A}$ and $\mathrm{B}$ of FDI as a function of the field strength $E_{0}$. The smallest strength of the laser field we consider in this figure is $E_{0}=0.02$ a.u.

possible that our model overestimates the DI probability for high field strengths; see discussion for Fig. 2. Indeed, when we consider a smaller peak field strength of 0.2 a.u., we find that the intensity-averaged KER distributions for FDI and DI, shown in Figs. 1(c) and 1(d), respectively, are both in better agreement with the experimental ones at higher intensity. We note that our results compare better with experiment than previously obtained classical results [11,12]. In those previous simulations the KER distributions for DI peak at considerably higher energies.

To find the upper limit of intensities that contribute to the KER distributions in Fig. 1, we analyze in Fig. 2 the FDI and DI probabilities as a function of the laser field strength. In this context, probability is the number of FDI or DI events relative to the number of initialized trajectories. At each intensity we ran enough trajectories to obtain at least 16000 FDI events and at least $50000 \mathrm{DI}$ events. Therefore, the statistical error of these results is very small.

Figure 2(a) shows that the DI probability increases quickly as a function of the field strength, already reaching a probability of $99.2 \%$ at a field strength of 0.38 a.u. Thus, all field strengths up to the peak intensity of 0.56 a.u. contribute significantly to the intensity-averaged KER distribution for DI in Fig. 1(b). On the other hand, Fig. 2 shows that the FDI probability reaches a maximum of $11.1 \%$ at an intermediate field strength of 0.08 a.u. and then decreases to $0.3 \%$ at a field strength of 0.56 a.u. Combined with the $1 / I$ factor in Eq. (3), we find that only field strengths up to roughly 0.32 a.u. contribute significantly to the intensity-averaged KER distribution for FDI in Fig. 1(b).

We now focus on describing in detail the FDI process for $\mathrm{D}_{3}{ }^{+}$. Similar to the case of $\mathrm{H}_{2}$ in [5], we identify two pathways that can lead to FDI. In the following we refer to the initially tunnel-ionized electron as electron 1 and to the initially bound electron as electron 2. In pathway A, electron 1 escapes while electron 2 tunnel ionizes later, while the laser field is on, and is eventually recaptured to a highly excited state of a $\mathrm{D}$ atom. In pathway B, electron 1 is eventually recaptured to a highly excited state of $\mathrm{D}$, while electron 2 tunnel ionizes later but eventually escapes. We find that the distribution of the internuclear distances at the time electron 2 tunnel ionizes peaks at around 3 a.u. for $\mathrm{D}_{3}{ }^{+}$. It is mainly after electron 2 tunnel ionizes that the nuclei rapidly dissociate, since tunnel ionization of electron 2 reduces the screening of the nuclei. It follows that the KER distribution for FDI should peak at 3 (number of nuclei)/3 (most probable nuclear distance for
Coulomb explosion) a.u., which is $27.2 \mathrm{eV}$. Indeed, we find the peak of the computed KER distribution for FDI to be around $21 \mathrm{eV}$. This value is smaller than $27 \mathrm{eV}$, as expected since one electron in FDI events remains bound, screening the Coulomb repulsion of the nuclei.

In Fig. 2(b) we show that the probability of pathway B of FDI reaches a maximum of $6.9 \%$ at a field strength of 0.14 a.u. and then decreases fast, reaching less than $1 \%$ at a field strength of 0.2 a.u. The dominance of pathway $\mathrm{B}$ at intermediate intensities is due to electron-electron correlation being much more prominent for these intensities [29]. Electron-electron correlation was shown to be more important for pathway $\mathrm{B}$ compared to pathway A also for strongly driven $\mathrm{H}_{2}$ [5]. This is to be expected, since in pathway B electron 1 , following tunnel ionization, later returns to the ion and interacts with electron 2. In addition, we find that, for $\mathrm{D}_{3}{ }^{+}$, at high intensities, the probability for pathway A of FDI decreases at a much slower rate than the probability of pathway B [see Fig. 2(b)]. The reason is that in pathway A electron 2 tunnels after gaining energy in a frustrated enhanced ionization process, i.e., electron 2 gains energy from the field in the same way as in an enhanced ionization process $[23,24]$ but electron 2 eventually does not escape. For higher intensities electron correlation plays a less important role compared to enhanced ionization. Hence, the probability for pathway A reduces at a smaller rate than the probability for B.

Next, we will identify the prevalent ionization mechanism leading to FDI in $\mathrm{D}_{3}{ }^{+}$. Specifically, we determine the probability of over-the-barrier ionization, $\mathrm{P}_{\mathrm{OBI}}$. In our notation $\mathrm{P}_{\mathrm{OBI}}$ not only refers to the permanent ionization of electron 2 in pathway $\mathrm{B}$ but also includes the temporary ionization of electron 2 in pathway A before it is being recaptured into an excited $\mathrm{D}^{*}$ state. We find that $\mathrm{P}_{\mathrm{OBI}}$ is around $9 \%$ for a field strength of 0.08 a.u., increasing to $87 \%$ at 0.56 a.u. However, for field strengths above 0.32 a.u., the probability of FDI events reduces significantly [see Fig. 2(a)]. Therefore, after integrating over field strengths up to 0.56 a.u., using Eq. (3), we find that over-the-barrier ionization accounts for $21 \%$ of FDI events. Thus, tunnel ionization dominates FDI.

Finally, we identify a feature of the break-up dynamics of the strongly driven triatomic that is a signature of pathway $\mathrm{B}$ and can be potentially observed experimentally. In Fig. 3 (a)/(b) we plot for FDI/DI the angle $\chi$ of the velocity of $\mathrm{D}^{*} / \mathrm{D}^{+}$with respect to the laser field for a field strength of 0.08 a.u. for the two alignments of the molecule with respect to the laser field considered. We find that, as for DI, for FDI the angular distribution has a three-lobe structure. The three-lobe structure we obtain for DI is in agreement with previous experiments [7]. For FDI we cannot provide a direct comparison with experiment, since the analyzed data in [7] includes the angular distribution of all single ionization events $\mathrm{D}^{+}+\mathrm{D}^{+}+\mathrm{D}$, i.e., FDI events as well as bond-softening events that yield the low KER peak in Figs. 1(a) and 1(c). Unlike DI, we find that the three lobes in Fig. 3(a) do not have equal weight, as is the case for DI in Fig. 3(b). Specifically, the lobe around $0^{\circ}$ has a $2 \%$ higher weight than the other two lobes for perpendicular alignment, and the lobe around $90^{\circ}$ has $2 \%$ less weight than the other two lobes for parallel alignment in Fig. 3(a). With respect to the initial state geometry of the nuclei of $\mathrm{D}_{3}{ }^{+}$in Fig. 3(c), this means that the electron that finally 


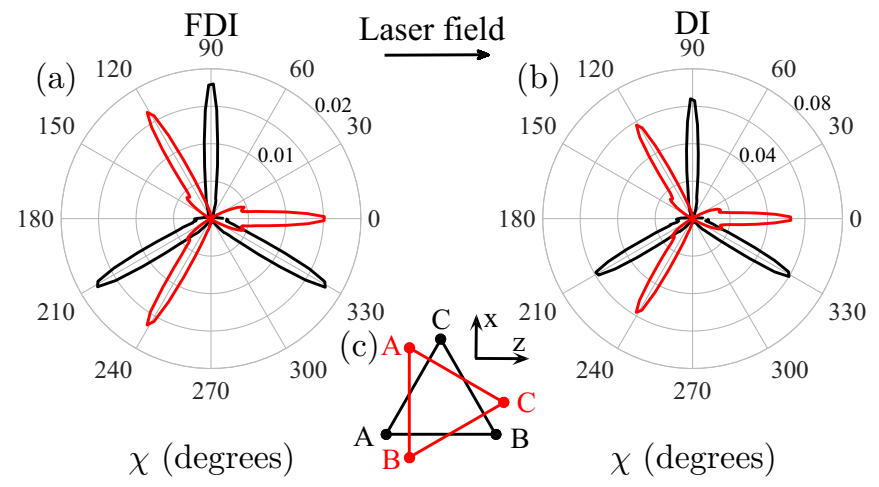

FIG. 3. The angle of the velocity vector of $\mathrm{D}^{*}$ in FDI (a) and of $\mathrm{D}^{+}$in DI (b) with respect to the laser field for parallel (black) and for perpendicular (red) alignment. The field strength is 0.08 a.u. (c) The initial state geometric configuration of $\mathrm{D}_{3}{ }^{+}$with respect to the laser field.

stays bound in FDI gets attached for parallel alignment more to either nucleus $\mathrm{A}$ or $\mathrm{B}$ rather than $\mathrm{C}$, while for perpendicular alignment to nucleus $\mathrm{C}$. This difference is reasonable, since frustrated enhanced ionization takes place mainly between the nuclei that are more parallel to the field, A and B for parallel alignment and $\mathrm{A}$ and $\mathrm{C}$ or $\mathrm{B}$ and $\mathrm{C}$ for perpendicular alignment. We find that this small difference in the weight of the lobes is present in both pathways A and B.

We find that the probability of the electron that finally remains bound in FDI to get attached to different nuclei varies significantly as a function of the initial velocity of the tunneling electron. For pathway A as a function of the initial velocity of electron 1, we find that the probability for electron 2 to get attached to nucleus $\mathrm{C}$ is between $1.5 \%$ and $2 \%$ smaller (larger) than the probability to get attached to nuclei A and $\mathrm{B}$ for parallel (perpendicular) alignment. Thus, the probability of electron 2 to get attached to nuclei $\mathrm{A}, \mathrm{B}$, and $\mathrm{C}$ is not sensitive to the initial velocity of electron 1 . However, we find that for pathway $\mathrm{B}$ the probability for electron 1 to get attached to nuclei $\mathrm{A}, \mathrm{B}$, and $\mathrm{C}$ varies with the initial velocity of electron 1 in the direction perpendicular to the field. Namely, for parallel alignment, we find that it is for small initial velocities of electron 1 that the probability of electron 1 to get attached to nucleus $\mathrm{C}$ differs the most from the probability of getting attached to nuclei $\mathrm{A}$ or $\mathrm{B}$; the probability to get attached to nucleus $\mathrm{C}$ is roughly $7 \%$ smaller. For perpendicular alignment, we find again that it is for small initial velocities of electron 1 that the probability of electron 1 to get attached to nucleus $\mathrm{C}$ differs the most from the probability of getting attached to nuclei $\mathrm{A}$ or $\mathrm{B}$; the probability to get attached to nucleus $\mathrm{C}$ is
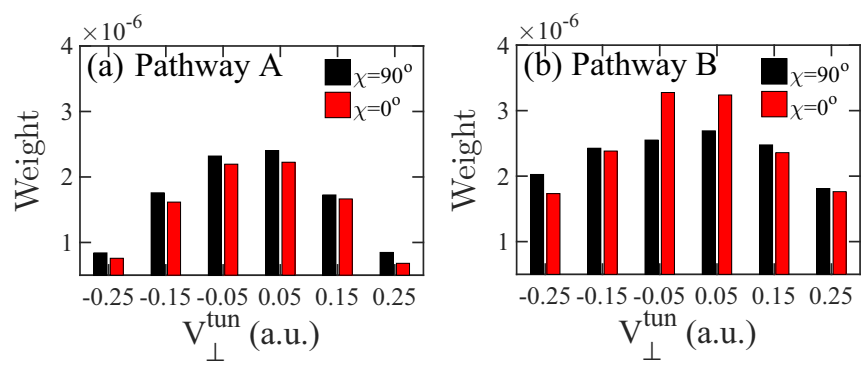

FIG. 4. The weight of the lobes around $0^{\circ}$ and $90^{\circ}$ for pathways A (a) and B (b).

roughly $7 \%$ larger. Expressing the above differences in terms of the lobes of the angular distribution of FDI, it means that, for pathway $\mathrm{B}$, for very small initial velocities of electron 1 the lobe around $90^{\circ}$ has $7 \%$ less weight than each of the other two lobes of parallel polarization, while the lobe around $0^{\circ}$ has $7 \%$ more weight than each of the other two lobes of perpendicular polarization. Thus, if the initial velocity of electron 1 can be probed experimentally, then one would observe a significant difference in the weight of the lobes around $0^{\circ}$ and $90^{\circ}$ that is due to pathway B. We illustrate the latter in Fig. 4. In Fig. 4(a), we show that in pathway $\mathrm{A}$ the difference in weight between the lobes around $0^{\circ}$ and $90^{\circ}$ is small and insensitive to the initial velocity of electron 1 . In contrast, in Fig. 4(b), we show that for pathway $\mathrm{B}$ the difference in weight between the lobes around $0^{\circ}$ and $90^{\circ}$ is very sensitive to the initial velocity of electron 1 and is large for small initial velocities.

\section{CONCLUSIONS}

Concluding, using a 3D semiclassical calculation where the Coulomb singularity is fully accounted for, we find that our results for the KER distribution for FDI of the strongly driven $\mathrm{D}_{3}{ }^{+}$agree well with experimental results. We also find that the underlying mechanism for FDI switches from tunnel to overthe-barrier ionization with increasing field strength. It would be interesting if future experiments identify the asymmetry in the angular distribution of the $\mathrm{D}^{*}$ fragment for FDI events, which we have shown to be a signature of pathway B of FDI.

\section{ACKNOWLEDGMENTS}

A.E. acknowledges I. Ben-Itzhak and J. McKenna for reanalyzing their published data in order to enable comparison with our simulations, as well as many discussions with I. BenItzhak. A.E. also acknowledges EPSRC Grant No. N031326/1 and the use of the computational resources of Legion at UCL.
[1] B. Manschwetus, T. Nubbemeyer, K. Gorling, G. Steinmeyer, U. Eichmann, H. Rottke, and W. Sandner, Phys. Rev. Lett. 102, 113002 (2009).

[2] T. Nubbemeyer, K. Gorling, A. Saenz, U. Eichmann, and W. Sandner, Phys. Rev. Lett. 101, 233001 (2008).

[3] T. Nubbemeyer, U. Eichmann, and W. Sandner, J. Phys. B 42, 134010 (2009); B. Manschwetus, H. Rottke, G. Steinmeyer, L. Foucar, A. Czasch, H. Schmidt-Böcking, and W. Sandner,
Phys. Rev. A 82, 013413 (2010); B. Ulrich, A. Vredenborg, A. Malakzadeh, M. Meckel, K. Cole, M. Smolarski, Z. Chang, T. Jahnke, and R. Dörner, ibid. 82, 013412 (2010).

[4] J. Wu, A. Vredenborg, B. Ulrich, L. Ph. H. Schmidt, M. Meckel, S. Voss, H. Sann, H. Kim, T. Jahnke, and R. Dörner, Phys. Rev. Lett. 107, 043003 (2011).

[5] A. Emmanouilidou, C. Lazarou, A. Staudte, and U. Eichmann, Phys. Rev. A 85, 011402(R) (2012). 
[6] H. Price, C. Lazarou, and A. Emmanouilidou, Phys. Rev. A 90, 053419 (2014).

[7] J. McKenna, A. M. Sayler, B. Gaire, N. G. Johnson, K. D. Carnes, B. D. Esry, and I. Ben-Itzhak, Phys. Rev. Lett. 103, 103004 (2009).

[8] J. Yao, B. Zeng, H. Xu, G. Li, W. Chu, J. Ni, H. Zhang, S. L. Chin, Y. Cheng, and Z. Xu, Phys. Rev. A 84, 051802(R) (2011).

[9] J. McKenna, A. M. Sayler, B. Gaire, N. G. Kling, B. D. Esry, K. D. Carnes, and I. Ben-Itzhak, New J. Phys. 14, 103029 (2012).

[10] A. M. Sayler, J. McKenna, B. Gaire, N. G. Kling, K. D. Carnes, and I. Ben-Itzhak, Phys. Rev. A 86, 033425 (2012).

[11] E. Lötstedt, T. Kato, and K. Yamanouchi, Phys. Rev. Lett. 106, 203001 (2011).

[12] E. Lötstedt, T. Kato, and K. Yamanouchi, J. Phys. B: At., Mol. Opt. Phys. 46, 235601 (2013).

[13] C. Lefebvre, H. Z. Lu, S. Chelkowski, and A. D. Bandrauk, Phys. Rev. A 89, 023403 (2014).

[14] V. G. Anicich and J. H. Futrell, Int. J. Mass Spectrom. Ion Processes 55, 189 (1984).

[15] T. Ergler, B. Feuerstein, A. Rudenko, K. Zrost, C. D. Schröter, R. Moshammer, and J. Ullrich, Phys. Rev. Lett. 97, 103004 (2006).

[16] E. Goll, G. Wunner, and A. Saenz, Phys. Rev. Lett. 97, 103003 (2006).

[17] D. Talbi and R. P. Saxon, J. Chem. Phys. 89, 2235 (1988).

[18] H.-J. Werner, P. J. Knowles, G. Knizia, F. R. Manby, M. Schütz et al., MOLPRO, version 2009, A package of ab initio programs (2009), see http://www.molpro.net.
[19] R. Murray, M. Spanner, S. Patchkovskii, and M. Yu. Ivanov, Phys. Rev. Lett. 106, 173001 (2011).

[20] M. V. Ammosov, N. B. Delete, and V. P. Krainov, Sov. Phys. JETP 64, 1191 (1986).

[21] L. Fechner, N. Camus, J. Ullrich, T. Pfeifer, and R. Moshammer, Phys. Rev. Lett. 112, 213001 (2014).

[22] C. Lazarou, A. Chen, and A. Emmanouilidou, arXiv:1511.05455.

[23] H. Niikura, F. Légaré, R. Hasbani, A. D. Bandrauk, M. Yu. Ivanov, D. M. Villeneuve, and P. B. Corkum, Nature (London) 417, 917 (2002).

[24] T. Zuo and A. D. Bandrauk, Phys. Rev. A 52, R2511(R) (1995); T. Seideman, M. Yu. Ivanov, and P. B. Corkum, Phys. Rev. Lett. 75, 2819 (1995); D. M. Villeneuve, M. Yu. Ivanov, and P. B. Corkum, Phys. Rev. A 54, 736 (1996); E. Dehghanian, A. D. Bandrauk, and G. L. Kamta, ibid. 81, 061403(R) (2010).

[25] P. Wang, A. M. Sayler, K. D. Carnes, B. D. Esry, and I. BenItzhak, Opt. Lett. 30, 664 (2005).

[26] F. Lindner, G. G. Paulus, H. Walther, A. Baltuška, E. Goulielmakis, M. Lezius, and F. Krausz, Phys. Rev. Lett. 92, 113001 (2004).

[27] C. Altucci, V. Tosa, R. Velotta, and C. H. Nam, Phys. Rev. A 70, 065402 (2004).

[28] P. H. Bucksbaum, A. Zavriyev, H. G. Muller, and D. W. Schumacher, Phys. Rev. Lett. 64, 1883 (1990).

[29] A. Emmanouilidou and A. Staudte, Phys. Rev. A 80, 053415 (2009). 